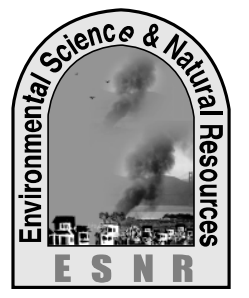

J. Environ. Sci. \& Natural Resources, 6(2):101-108, 2013

ISSN 1999-7361

\title{
Growth Parameters of Transplant Aman Rice (cv. BRRI dhan 52) as Influenced by Age of Tiller Seedlings, Number of Tiller Seedlings Hill $^{-1}$ and Level of USG
}

\author{
K. S. Rahman, S. K. Paul, M. A. R. Sarkar and M. S. Islam
}

Department of Agronomy, Bangladesh Agricultural University, Mymensingh-2202, Bangladesh

\begin{abstract}
The experiment was conducted at the Agronomy Field Laboratory, Bangladesh Agricultural University, Mymensingh, Bangladesh to investigate the effect of age of tiller seedlings, number of tiller seedlings hill ${ }^{-1}$ and application of urea super granules (USG) on the growth parameters of transplant aman rice cv. BRRI dhan52. The experiment comprised two ages of tiller seedlings viz. 25 and 35 days old, three levels of tiller seedling hill ${ }^{-1}$ viz. 1,3 and 5 seedlings hill $^{-1}$ and three USG levels viz. $0,1.8$ and 2.7g USG. The experiment was laid out in a Randomized Complete Block Design with three replications. The highest and lowest plant height was found by transplanting 25-day and 35-day old tiller seedlings at all dates of observations. By transplanting 35-day old tiller seedlings total dry matter hill ${ }^{-1}$ was found highest at 15DAT and 45 DAT. Total dry matter hill ${ }^{-1}$ was the highest in 5 tiller seedlings hill ${ }^{-1}$ and it was in lowest in 1 tiller seedling hill ${ }^{-1}$. Plant height was the highest in 5 tiller seedlings hill ${ }^{-1}$ which was as good as that of 3 tiller seedlings hill ${ }^{-1}$ and the lowest one was found in 1 tiller seedling hill ${ }^{-1}$ at 15 DAT. The tallest plant and highest total dry matter hill ${ }^{-1}$ were observed in 1.8g USG and the lowest one was observed in control treatment. Transplant Aman rice can be grown by transplanting 25 -day old tiller seedling, 5 tiller seedlings hill ${ }^{-1}$ and by applying $1.8 \mathrm{~g}$ USG for the highest plant height, more tiller and total dry matter production hill ${ }^{-1}$.
\end{abstract}

Key words: Growth, Transplant Aman rice, Tiller seedling, USG

\section{Introduction}

Bangladesh is a flood prone country. Devastating flood very often washes away transplant Aman rice in Bangladesh. Farmers cannot re-transplant the affected land in the early and mid-September due to unavailability of seedlings. If available, seedlings are either too young or too old to produce a good crop. Thus limitation of seedlings is a great problem in this situation. Re-transplantation of separated tillers from an unaffected Aman crop may be used as seedlings to rehabilitate the damaged rice. Double transplanting practices has also been suggested for transplant Aman areas where transplanting is delayed due to flood water inundation (Alim et al. 1962). Rice has unique ability to tiller profusely as each leaf axils has the potential to produce a tiller. In rice, many of the late tillers do not produce due to higher population. This technique of transplanting of separated tillers may be a promising alternative for growing a post flood transplant aman crop (Sarkar et al., 2011; Mridha et al., 1991; Siddique et al., 1991). Biswas and Salokhe (2001) suggested that in some flood-prone lowlands, where the transplanted crop is damaged by natural hazard, vegetative propagation using tiller separated (4 tillers hill $^{-1}$ ) from the previously established transplanted crop gave higher yield than nursery seedlings transplanted on the same date. Paul et al. (2002) reported that tillers can be separated at 25 or 35 days after transplanting (DAT) without hampering grain yield. In flood-prone areas, where seedlings become unavailable for transplanting due to early or late floods, transplanting 25-days old tiller seedlings with 2 tiller seedlings hill $^{-1}$ improved yield of transplant Aman rice (Sarkar et al., 2011). Removal of some tillers from the mother hill may help better development of the remaining tillers. Separated tillers can be used as tiller seedlings to replant especially in post flood situation.

\section{Materials and Methods}

The experiment was conducted at the Agronomy field laboratory, Bangladesh Agricultural University, Mymensingh, during the period from July to December 2012 to study the influence of age of tiller seedlings, number of tiller seedlings hill ${ }^{-1}$ and urea super granules (USG) on growth of transplant aman rice. The experimental sites belongs to the Sonatola Soil Series of Old Brahmaputra Floodplain (AEZ 9) having non calcareous dark grey floodplain soil. The land was medium high with sandy loam texture having $\mathrm{pH}$ 5.9. BRRI dhan52, a high yielding modern rice variety of transplant Aman rice, developed by the Bangladesh Rice Research Institute, has been used as the test crop. The experiment comprised two ages of tiller seedlings viz. 25 and 35 days old, three levels of tiller seedling hill ${ }^{-1}$ viz.1, 3 and 5 seedlings hill ${ }^{-1}$ and three USG levels viz. 0, 1.8 and $2.7 \mathrm{~g}$ USG. The experiment was laid out in a Randomized Complete Block Design with three replications. Each block was divided into 18 unit plots where 18 treatment combinations were allocated at random. The distance between the unit plots and block were $0.5 \mathrm{~m}$ and $1 \mathrm{~m}$ respectively. There were 54 unit plots in the experiment. The size of unit plot was $4 \mathrm{~m} \times 2.5 \mathrm{~m}$. The experimental plots were fertilized with triple super phosphate (TSP), muriate of potash (MoP), gypsum and zinc sulphate @ 100,70,60,10 kg ha ${ }^{-1}$. The entire amounts of triple super phosphate (TSP), 
muriate of potash (MoP), and gypsum and zinc sulphate were applied at final land preparation. Nitrogen was applied according to experimental specification in the form of urea super granules (USG) at 10 days after transplanting 4 hill $^{-1}$ in every alternate row. Four hills were randomly selected and marked with the bamboo sticks in each unit plot excluding border rows to record the data on plant height and tiller number. Plant height, tiller number, total dry matter were recorded four times at 15, 30, 45, 55 and 75 days after transplanting (DAT). For total dry matter determination, four hills were randomly selected in each plot excluding border rows. The plant samples were packed in labeled brown paper bags and dried in the oven at $80 \pm 5^{\circ} \mathrm{C}$ for 72 hours until constant weight was reached. Recorded data were analyzed statistically using "Analysis of Variance Technique and the differences among treatment means were adjudged by Duncan's Multiple Range Test (DMRT).

\section{Results and Discussion}

\section{Plant height}

Plant height was significantly affected by age of tiller seedlings during at 15, 30, 45, 60 and 75 days after transplanting (DAT). The highest plant height was obtained at all sampling dates when 25-day old tiller seedling was transplanted compared to transplanting older seedling (35days old). Similar results were obtained by Hossain et al. (2011). This reduction in plant height was mainly due to the availability of short vegetative growth period for 35-day old tiller seedlings in comparison with 25-day old tiller seedling. Separation of tiller at 35 DAT resulted in reduced plant height where tiller separation at 25 DAT produced taller plants. Molla et al. (1992) reported that late tiller separation reduced plant height significantly to early tiller separation (Figure 1). Plant height was significantly affected by number of tiller seedlings hill $^{-1}$ within the period of 15,45 and 60 DAT. The highest plant height was obtained at 60 DAT when 1 tiller seedling were transplanted hill ${ }^{-1}$ and the lowest plant height was found during at 45 DAT when 5 tiller seedlings were transplanted hill $^{-1}$ (Table 1). Plant height was significantly affected by USG application at 15, 30, 45 and 75 DAT. At all sampling dates the highest plant height was found when $1.8 \mathrm{~g}$ USG was applied and the lowest plant height was found when USG was not applied (Table 1).
Plant height was significantly influenced by the interaction between the age of tiller seedlings and number of tiller seedlings hill ${ }^{-1}$ age of tiller seedlings and USG application number of tiller seedlings hill $^{-1}$ and USG application, age of tiller seedlings, number of tiller seedlings hill ${ }^{-1}$ and USG application at various dates of sampling. At 15 DAT the highest plant height was found in 35-day old tiller seedlings by transplanting 3 tiller seedlings hill $^{-1}$ and the lowest plant height was found in 25-day old tiller seedlings by transplanting 1 tiller seedling hill ${ }^{-1}$. At 45 DAT the highest plant height was found in 35-day old tiller seedlings by transplanting 5 tiller seedlings hill ${ }^{-1}$ and the lowest plant height was found in 25-day old tiller seedlings by transplanting 1 tiller seedlings hill ${ }^{-1}$. At 60 DAT the highest Plant height was found in 35-day old tiller seedlings by transplanting 3 tiller seedlings hill ${ }^{-1}$ and the lowest plant height was found in 25-day old tiller seedlings by transplanting 3 tiller seedlings hill ${ }^{-1}$ (Table 2). The highest plant height was found in 25-day old tiller seedlings when $1.8 \mathrm{~g}$ USG was applied and the lowest plant height was found in 25-day old tiller seedlings when USG was not applied (Table 3). At 45 DAT significantly influenced on plant height. The highest plant height was found by transplanting 5 tiller seedlings hill $^{-1}$ when $1.8 \mathrm{~g}$ USG was applied and the lowest plant height was found by transplanting 1 tiller seedling hill ${ }^{-1}$ when USG was not applied. At 60 DAT the highest plant height was found by transplanting 1 tiller seedling hill ${ }^{-1}$ when $1.8 \mathrm{~g}$ USG was applied and the lowest plant height was found by transplanting 3 tiller seedlings hill ${ }^{-1}$ when $2.7 \mathrm{~g}$ USG was applied. At 75 DAT the highest plant height was found by transplanting 3 tiller seedlings hill ${ }^{-1}$ when $1.8 \mathrm{~g} \mathrm{USG}$ was applied and the lowest plant height was found by transplanting 1 tiller seedling hill ${ }^{-1}$ when USG was not applied (Table 4). At 15 DAT the highest plant height was found in 35-day old tiller seedling by transplanting 5 tiller seedlings hill ${ }^{-1}$ with the application of $1.8 \mathrm{~g} \mathrm{USG}$ and the lowest plant height was found in 25-day old tiller seedlings by transplanting 3 tiller seedlings hill ${ }^{-}$ ${ }^{1}$ without application of USG. At 45 DAT the highest plant height was found in 35-day old tiller seedling by transplanting 5 tiller seedlings hill $^{-1}$ with the application of $1.8 \mathrm{~g}$ USG and the lowest plant height was found in 25-day old tiller seedlings by transplanting 1 tiller seedling hill ${ }^{-1}$ without application of USG (Table 5). 
Table 1. Effect of age of tiller seedlings, number of tiller seedlings hill ${ }^{-1}$ and USG application on plant height, number of tiller hill ${ }^{-1}$ and total dry matter hill ${ }^{-1}$

\begin{tabular}{|c|c|c|c|c|c|c|c|c|c|c|c|c|c|c|c|}
\hline & \multicolumn{5}{|c|}{ Plant height $(\mathrm{cm})$} & \multicolumn{5}{|c|}{ Number of tiller hill ${ }^{-1}$} & \multicolumn{5}{|c|}{ Total dry matter hill ${ }^{-1}(\mathrm{~g})$} \\
\hline & \multicolumn{5}{|c|}{ Days after transplanting } & \multicolumn{5}{|c|}{ Days after transplanting } & \multicolumn{5}{|c|}{ Days after transplanting } \\
\hline & 15 & 30 & 45 & 60 & 75 & 15 & 30 & 45 & 60 & 75 & 15 & 30 & 45 & 60 & 75 \\
\hline \multicolumn{16}{|c|}{ Age of tiller seedling } \\
\hline $\mathrm{A}_{1}$ & $54.64 \mathrm{a}$ & $67.95 \mathrm{a}$ & $78.27 \mathrm{a}$ & $79.06 \mathrm{a}$ & $107.31 \mathrm{a}$ & 4.84 & 6.70 & 8.43 & 10.65 & 12.97 & $0.91 b$ & 2.29 & $3.24 \mathrm{~b}$ & 6.62 & $10.26 \mathrm{a}$ \\
\hline $\mathrm{A}_{2}$ & $50.42 \mathrm{~b}$ & $65.90 \mathrm{~b}$ & $74.81 \mathrm{~b}$ & $77.78 \mathrm{~b}$ & $106.09 \mathrm{~b}$ & 4.65 & 6.51 & 8.22 & 10.47 & 12.79 & $1.09 \mathrm{a}$ & 2.34 & $3.34 \mathrm{a}$ & 6.49 & $9.70 \mathrm{~b}$ \\
\hline \multicolumn{16}{|c|}{ No. of tiller seedlings hill ${ }^{-1}$} \\
\hline $\mathrm{T}_{1}$ & $50.10 \mathrm{~b}$ & 66.73 & $74.91 \mathrm{~b}$ & $80.14 a$ & 106.39 & 4.61 & 6.40 & 8.71 & 10.46 & 12.91 & 0.92 & $2.06 \mathrm{c}$ & $3.03 \mathrm{c}$ & $6.36 \mathrm{c}$ & $9.35 \mathrm{c}$ \\
\hline $\mathrm{T}_{2}$ & $53.23 \mathrm{a}$ & 65.86 & $78.96 \mathrm{a}$ & $76.71 \mathrm{c}$ & 106.62 & 4.55 & 6.35 & 8.66 & 10.41 & 12.86 & 0.96 & $2.35 \mathrm{~b}$ & $3.33 \mathrm{~b}$ & $6.40 \mathrm{~b}$ & $9.75 b$ \\
\hline $\mathrm{T}_{3}$ & $54.26 \mathrm{a}$ & 68.19 & $75.77 \mathrm{~b}$ & $78.41 \mathrm{~b}$ & 107.10 & 4.66 & 6.46 & 8.77 & 10.52 & 12.97 & 1.13 & $2.54 \mathrm{a}$ & $3.52 \mathrm{a}$ & $6.90 \mathrm{a}$ & $10.85 \mathrm{a}$ \\
\hline \multicolumn{16}{|c|}{ USG application } \\
\hline $\mathrm{N}_{0}$ & $51.86 \mathrm{~b}$ & $65.54 \mathrm{~b}$ & $73.32 \mathrm{c}$ & 77.96 & $104.69 \mathrm{c}$ & 4.50 & 6.45 & 8.73 & 10.42 & 12.87 & 1.07 & 2.40 & 3.37 & $6.23 \mathrm{c}$ & $9.02 \mathrm{c}$ \\
\hline $\mathrm{N}_{1}$ & $54.58 \mathrm{a}$ & $68.87 \mathrm{a}$ & $79.67 \mathrm{a}$ & 79.19 & $108.29 \mathrm{a}$ & 4.66 & 6.40 & 8.68 & 10.37 & 12.82 & 1.04 & 2.33 & 3.31 & $6.88 \mathrm{a}$ & $10.75 \mathrm{a}$ \\
\hline $\mathrm{N}_{2}$ & $51.15 \mathrm{~b}$ & $66.37 \mathrm{~b}$ & $76.65 \mathrm{~b}$ & 78.10 & $107.13 b$ & 4.66 & 6.51 & 8.79 & 10.48 & 12.93 & 0.89 & 2.23 & 3.19 & $6.55 \mathrm{~b}$ & $10.18 \mathrm{~b}$ \\
\hline
\end{tabular}

Figures in column under each factor of treatment having the same letter or without letter do not differ significantly whereas figures with dissimilar letter(s) differ significantly as per DMRT, $A_{1}=25$-day old tiller seedlings, $A_{2}=35$-day old tiller seedlings, $\mathrm{T}_{1}=1$ tiller seedlings hill ${ }^{-1}, \mathrm{~T}_{2}=3$ tiller seedlings hill $^{-1}, \mathrm{~T}_{3}=5$ tiller seedlings hill $^{-1}, \mathrm{~N}_{0}=\mathrm{control}, \mathrm{N}_{1}=$ $1.8 \mathrm{~g} \mathrm{USG}, \mathrm{N}_{2}=2.7 \mathrm{~g}$ USG.

Table 2. Interaction effect of age of tiller seedlings and number of tiller seedlings hill ${ }^{-1}$ on plant height, number of tiller hill ${ }^{-1}$ and total dry matter hill ${ }^{-1}$

\begin{tabular}{|c|c|c|c|c|c|c|c|c|c|c|c|c|c|c|c|c|}
\hline \multirow{3}{*}{$\begin{array}{l}\text { Age of tiller } \\
\text { seedlings } \\
\text { (days) }\end{array}$} & \multirow{3}{*}{$\begin{array}{l}\text { No. } \\
\text { of } \\
\text { tiller } \\
\text { seed } \\
\text { lings } \\
\text { hill }^{-1}\end{array}$} & \multicolumn{5}{|c|}{ Plant height $(\mathrm{cm})$} & \multicolumn{5}{|c|}{ Number of tillers } & \multicolumn{5}{|c|}{ Total dry matter hill $^{-1}(\mathrm{~g})$} \\
\hline & & \multicolumn{5}{|c|}{ Days after transplanting } & \multicolumn{5}{|c|}{ Days after transplanting } & \multicolumn{5}{|c|}{ Days after transplanting } \\
\hline & & 15 & 30 & 45 & 60 & 75 & 15 & 30 & 45 & 60 & 75 & 15 & 30 & 45 & 60 & 75 \\
\hline \multirow[t]{3}{*}{$\mathrm{A}_{1}$} & $\mathrm{~T}_{1}$ & $50.20 \mathrm{~b}$ & 65.72 & $73.31 \mathrm{c}$ & $76.36 \mathrm{~b}$ & 105.36 & $4.78 \mathrm{ab}$ & $6.78 \mathrm{ab}$ & $8.67 \mathrm{ab}$ & $10.44 \mathrm{ab}$ & 12.67 & $0.85 \mathrm{~b}$ & 2.15 & 3.03 & 6.51 & 9.83 \\
\hline & $\mathrm{T}_{2}$ & $50.79 b$ & 65.57 & $75.17 \mathrm{bc}$ & $77.06 \mathrm{~b}$ & 106.30 & $4.00 \mathrm{~b}$ & $6.00 \mathrm{~b}$ & $7.44 \mathrm{~d}$ & $9.33 \mathrm{c}$ & 11.89 & $0.89 \mathrm{~b}$ & 2.15 & 3.12 & 6.46 & 10.19 \\
\hline & $\mathrm{T}_{3}$ & $50.25 b$ & 66.40 & $75.96 b$ & $78.67 b$ & 106.61 & $4.44 \mathrm{ab}$ & $6.44 \mathrm{ab}$ & $8.11 \mathrm{bcd}$ & $9.89 \mathrm{abc}$ & 12.89 & $0.99 b$ & 2.58 & 3.58 & 6.89 & 10.78 \\
\hline \multirow[t]{3}{*}{$\mathrm{A}_{2}$} & $\mathrm{~T}_{1}$ & $50.00 \mathrm{~b}$ & 67.73 & $76.50 \mathrm{~b}$ & $78.13 b$ & 107.41 & $4.00 \mathrm{~b}$ & $6.00 \mathrm{~b}$ & $7.78 \mathrm{~cd}$ & $9.78 \mathrm{bc}$ & 12.78 & $0.85 \mathrm{~b}$ & 1.98 & 3.02 & 6.20 & 8.88 \\
\hline & $\mathrm{T}_{2}$ & $55.67 \mathrm{a}$ & 66.14 & $76.37 \mathrm{~b}$ & $82.15 \mathrm{a}$ & 106.94 & $5.00 \mathrm{a}$ & $7.00 \mathrm{a}$ & $9.00 \mathrm{a}$ & $10.67 \mathrm{a}$ & 13.00 & $1.03 \mathrm{~b}$ & 2.55 & 3.55 & 6.35 & 9.32 \\
\hline & $\mathrm{T}_{3}$ & $58.27 \mathrm{a}$ & 69.98 & $81.96 \mathrm{a}$ & $78.14 \mathrm{~b}$ & 107.59 & $4.56 \mathrm{ab}$ & $6.56 \mathrm{ab}$ & $8.56 \mathrm{abc}$ & $10.33 \mathrm{ab}$ & 13.11 & $1.40 \mathrm{a}$ & 2.50 & 3.46 & 6.92 & 10.92 \\
\hline \multirow{2}{*}{$\mathrm{CV}(\%)$} & & & & & & & & & & & & & & 11.7 & & \\
\hline & & 5.44 & 5.72 & 3.12 & 3.63 & 3.62 & 12.19 & 12.56 & 9.66 & 7.98 & 6.46 & 9.69 & 16.96 & 2 & 9.7 & 8.12 \\
\hline $\begin{array}{l}\text { Level of } \\
\text { significance }\end{array}$ & & $* *$ & NS & * & * & NS & $* *$ & $* *$ & $* *$ & $* *$ & NS & $* *$ & NS & NS & NS & NS \\
\hline
\end{tabular}

Figures in column under each factor of treatment having the same letter or without letter do not differ significantly whereas figures with dissimilar letter(s) differ significantly as per DMRT, $* *=$ Significant at $1 \%$ level of probability, $*=$ Significant at $5 \%$ level of probability, NS $=$ Not significant, $A_{1}=25$-day old tiller seedlings, $A_{2}=35$-day old tiller seedlings,

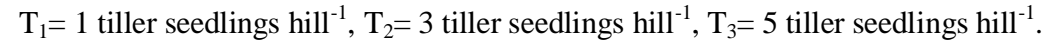


Table 3. Interaction effect of age of tiller seedlings and USG application on plant height, number of tiller hill ${ }^{-1}$ and total dry matter hill ${ }^{-1}$

\begin{tabular}{|c|c|c|c|c|c|c|c|c|c|c|c|c|c|c|c|c|}
\hline \multirow{3}{*}{$\begin{array}{c}\text { No. of } \\
\text { tiller } \\
\text { seedlings } \\
\text { hill- }^{-1}\end{array}$} & \multirow{3}{*}{$\begin{array}{c}\text { USG } \\
\text { applicati } \\
\text { on }\end{array}$} & \multicolumn{5}{|c|}{ Plant height $(\mathrm{cm})$} & \multicolumn{5}{|c|}{ Number of tillers } & \multicolumn{5}{|c|}{ Total dry matter hill ${ }^{-1}(\mathrm{~g})$} \\
\hline & & \multicolumn{5}{|c|}{ Days after transplanting } & \multicolumn{5}{|c|}{ Days after transplanting } & \multicolumn{5}{|c|}{ Days after transplanting } \\
\hline & & 15 & 30 & 45 & 60 & 75 & 15 & 30 & 45 & 60 & 75 & 15 & 30 & 45 & 60 & 75 \\
\hline \multirow[t]{3}{*}{$\mathrm{A}_{1}$} & $\mathrm{~N}_{0}$ & 49.55 & $63.07 \mathrm{c}$ & $69.79 \mathrm{~d}$ & 78.14 & $103.81 \mathrm{c}$ & $3.44 d$ & $5.44 d$ & $6.56 \mathrm{~d}$ & $7.89 \mathrm{~d}$ & $9.67 \mathrm{~d}$ & 1.05 & 2.37 & 3.33 & $6.13 b$ & $9.09 \mathrm{~b}$ \\
\hline & $\mathrm{N}_{1}$ & 53.08 & $69.89 \mathrm{a}$ & $80.29 \mathrm{a}$ & 80.13 & $108.96 \mathrm{a}$ & $5.44 \mathrm{a}$ & $7.44 \mathrm{a}$ & $9.33 \mathrm{a}$ & $11.67 \mathrm{a}$ & $14.67 \mathrm{a}$ & 0.95 & 2.38 & 3.36 & $6.70 \mathrm{ab}$ & 10.48 \\
\hline & $\mathrm{N}_{2}$ & 48.62 & $64.73 b c$ & $74.37 \mathrm{c}$ & 78.90 & $105.50 \mathrm{~b}$ & $4.33 b c$ & $6.33 b c$ & $8.33 b c$ & $10.11 \mathrm{~b}$ & $13.11 \mathrm{~b}$ & 0.74 & 2.13 & 3.04 & $7.03 \mathrm{a}$ & $11.28 \mathrm{a}$ \\
\hline \multirow[t]{3}{*}{$\mathrm{A}_{2}$} & $\mathrm{~N}_{0}$ & 54.18 & $68.00 \mathrm{ab}$ & $76.84 \mathrm{~b}$ & 77.79 & $105.58 \mathrm{~b}$ & $4.56 b c$ & $6.56 \mathrm{bc}$ & $7.78 \mathrm{c}$ & $9.22 \mathrm{c}$ & $11.67 \mathrm{c}$ & 1.10 & 2.42 & 3.42 & $6.32 \mathrm{~b}$ & $9.02 \mathrm{~b}$ \\
\hline & $\mathrm{N}_{1}$ & 56.08 & $67.86 \mathrm{ab}$ & 79.04ab & 78.24 & $107.61 \mathrm{a}$ & $4.00 \mathrm{~cd}$ & $6.00 \mathrm{~cd}$ & $8.78 \mathrm{ab}$ & $10.78 \mathrm{~b}$ & $13.78 \mathrm{~b}$ & 1.13 & 2.28 & 3.26 & $7.07 \mathrm{a}$ & $11.01 \mathrm{a}$ \\
\hline & $\mathrm{N}_{2}$ & 53.67 & $68.00 \mathrm{ab}$ & $78.93 \mathrm{ab}$ & 77.30 & $108.76 \mathrm{a}$ & $5.00 \mathrm{ab}$ & $7.00 \mathrm{ab}$ & $8.78 \mathrm{ab}$ & $10.78 \mathrm{~b}$ & $13.44 \mathrm{~b}$ & 1.05 & 2.33 & 3.35 & $6.08 \mathrm{~b}$ & $9.03 b$ \\
\hline $\mathrm{CV}(\%)$ & & 5.44 & 5.72 & 3.12 & 3.63 & 3.62 & 12.19 & 12.56 & 9.66 & 7.98 & 6.46 & 9.69 & 16.96 & 11.72 & 9.7 & 8.12 \\
\hline $\begin{array}{c}\text { Level of } \\
\text { significanc } \\
\mathrm{e}\end{array}$ & & NS & $* *$ & $* *$ & NS & $* *$ & $* *$ & $* *$ & $* *$ & $* *$ & $* *$ & NS & NS & NS & $* *$ & $* *$ \\
\hline
\end{tabular}

Figures in column under each factor of treatment having the same letter or without letter do not differ significantly whereas figures with dissimilar letter(s) differ significantly as per DMRT, $*=$ Significant at $5 \%$ level of probability, NS = Not significant, $A_{1}=25$-day old tiller seedlings, $A_{2}=35$-day old tiller seedlings, $N_{0}=$ control, $N_{1}=1.8 \mathrm{~g} \mathrm{USG}, \mathrm{N}_{2}=2.7 \mathrm{~g}$ USG

Table 4. Interaction effect of age of tiller seedlings and number of tiller seedlings hill ${ }^{-1}$ on plant height, number of tiller hill ${ }^{-1}$ and total dry matter hill ${ }^{-1}$

\begin{tabular}{|c|c|c|c|c|c|c|c|c|c|c|c|c|c|c|c|c|}
\hline \multirow{3}{*}{$\begin{array}{l}\text { Number of } \\
\text { tiller } \\
\text { seedlings } \\
\text { hilll }^{-1}\end{array}$} & \multirow{3}{*}{$\begin{array}{c}\text { USG } \\
\text { application }\end{array}$} & \multicolumn{5}{|c|}{ Plant height $(\mathrm{cm})$} & \multicolumn{5}{|c|}{ Number of tillers } & \multicolumn{5}{|c|}{ Total dry matter hill ${ }^{-1}(\mathrm{~g})$} \\
\hline & & \multicolumn{5}{|c|}{ Days after transplanting } & \multicolumn{5}{|c|}{ Days after transplanting } & \multicolumn{5}{|c|}{ Days after transplanting } \\
\hline & & 15 & 30 & 45 & 60 & 75 & 15 & 30 & 45 & 60 & 75 & 15 & 30 & 45 & 60 & 75 \\
\hline \multirow[t]{3}{*}{$\mathrm{T}_{1}$} & $\mathrm{~N}_{0}$ & 50.46 & 65.58 & $71.06 \mathrm{~d}$ & $76.91 \mathrm{bc}$ & $102.20 \mathrm{c}$ & 3.67 & $5.67 \mathrm{c}$ & $6.83 \mathrm{~d}$ & 8.50 & 10.33 & 1.11 & 2.13 & 3.07 & $5.35 \mathrm{~d}$ & $6.95 \mathrm{f}$ \\
\hline & $\mathrm{N}_{1}$ & 50.02 & 68.07 & $76.55 \mathrm{c}$ & $81.90 \mathrm{a}$ & $107.60 \mathrm{a}$ & 4.83 & $6.83 \mathrm{ab}$ & $9.17 \mathrm{ab}$ & 11.17 & 14.17 & 0.87 & 1.97 & 2.97 & $6.62 b c$ & $10.52 b c$ \\
\hline & $\mathrm{N}_{2}$ & 49.82 & 66.53 & $77.12 \mathrm{bc}$ & $81.62 \mathrm{a}$ & $108.20 \mathrm{a}$ & 4.67 & $6.67 \mathrm{abc}$ & $8.67 \mathrm{ab}$ & 10.67 & 13.67 & 0.78 & 2.09 & 3.04 & $6.00 \mathrm{~cd}$ & $9.28 \mathrm{de}$ \\
\hline \multirow[t]{3}{*}{$\mathrm{T}_{2}$} & $\mathrm{~N}_{0}$ & 50.97 & 63.76 & $71.41 \mathrm{~d}$ & $78.33 \mathrm{abc}$ & $103.72 b c$ & 4.50 & $6.50 \mathrm{abc}$ & $7.33 \mathrm{~cd}$ & 8.50 & 10.33 & 1.15 & 2.57 & 3.59 & $6.12 \mathrm{~cd}$ & $8.61 \mathrm{e}$ \\
\hline & $\mathrm{N}_{1}$ & 57.24 & 69.28 & $79.62 b$ & $76.35 \mathrm{bc}$ & $108.76 \mathrm{a}$ & 4.17 & $6.17 \mathrm{abc}$ & $8.50 \mathrm{ab}$ & 11.00 & 14.00 & 0.92 & 2.41 & 3.38 & $7.72 \mathrm{a}$ & $11.83 \mathrm{a}$ \\
\hline & $\mathrm{N}_{2}$ & 51.48 & 64.53 & $76.28 \mathrm{c}$ & $75.43 \mathrm{c}$ & $108.55 \mathrm{a}$ & 4.83 & $6.83 \mathrm{ab}$ & $8.83 \mathrm{ab}$ & 10.50 & 13.00 & 0.82 & 2.07 & 3.03 & $6.47 \mathrm{bc}$ & $10.12 \mathrm{~cd}$ \\
\hline \multirow[t]{3}{*}{$T_{3}$} & $\mathrm{~N}_{0}$ & 54.17 & 67.27 & $77.48 \mathrm{bc}$ & $78.65 \mathrm{abc}$ & $108.17 \mathrm{a}$ & 3.83 & $5.83 \mathrm{bc}$ & $7.33 \mathrm{~cd}$ & 8.67 & 11.33 & 0.96 & 2.49 & 3.46 & $7.22 \mathrm{ab}$ & $11.50 \mathrm{ab}$ \\
\hline & $\mathrm{N}_{1}$ & 56.48 & 69.27 & $82.83 \mathrm{a}$ & 79.32ab & $108.50 \mathrm{a}$ & 5.17 & $7.17 \mathrm{a}$ & $9.50 \mathrm{a}$ & 11.50 & 14.50 & 1.33 & 2.60 & 3.58 & $6.31 \mathrm{c}$ & $9.88 \mathrm{~cd}$ \\
\hline & & 52.13 & 68.03 & $76.55 \mathrm{c}$ & $77.25 \mathrm{bc}$ & $104.63 \mathrm{~b}$ & 4.50 & $6.50 \mathrm{abc}$ & $8.17 b c$ & 10.17 & 13.17 & 1.08 & 2.52 & 3.52 & $7.19 \mathrm{ab}$ & $11.16 \mathrm{ab}$ \\
\hline $\mathrm{CV}(\%)$ & & 5.44 & 5.72 & 3.12 & 3.63 & 3.62 & 12.19 & 12.56 & 9.66 & 7.98 & 6.46 & 9.69 & 16.96 & 11.72 & 9.7 & 8.12 \\
\hline $\begin{array}{c}\text { Level of } \\
\text { significance }\end{array}$ & & NS & NS & $* *$ & $* *$ & $* *$ & NS & $*$ & $* *$ & NS & NS & NS & NS & NS & $* *$ & $* *$ \\
\hline
\end{tabular}

Figures in column under each factor of treatment having the same letter or without letter do not differ significantly whereas figures with dissimilar letter(s) differ significantly as per DMRT, $* *=$ Significant at $1 \%$ level of probability, ${ }^{*}=$ Significant at $5 \%$ level of probability, NS $=$ Not significant, $\mathrm{T}_{1}=1$ tiller seedlings hill ${ }^{-1}, \mathrm{~T}_{2}=3$ tiller seedlings hill ${ }^{-1}, \mathrm{~T}_{3}=5$ tiller seedlings hill $^{-1}, \mathrm{~N}_{0}=$ control, $\mathrm{N}_{1}=1.8 \mathrm{~g}$ USG, $\mathrm{N}_{2}=2.7 \mathrm{~g}$ USG. 
Table 5. Interaction effect of age of tiller seedlings, number of tiller seedlings hill ${ }^{-1}$ and USG application on plant height, number of tiller hill ${ }^{-1}$ and total dry matter hill ${ }^{-1}$

\begin{tabular}{|c|c|c|c|c|c|c|c|c|c|c|c|c|c|c|c|c|c|}
\hline \multirow{3}{*}{$\begin{array}{l}\text { Age of } \\
\text { tiller } \\
\text { seedlings } \\
\text { (days) }\end{array}$} & \multirow{3}{*}{$\begin{array}{c}\text { Number of } \\
\text { tiller } \\
\text { seedlings } \\
\text { hill }^{-1} \\
\end{array}$} & \multirow{3}{*}{$\begin{array}{c}\text { USG } \\
\text { application }\end{array}$} & \multicolumn{5}{|c|}{ Plant height $(\mathrm{cm})$} & \multicolumn{5}{|c|}{ Number of tillers } & \multicolumn{5}{|c|}{ Total dry matter hill ${ }^{-1}(\mathrm{~g})$} \\
\hline & & & \multicolumn{5}{|c|}{ Days after transplanting } & \multicolumn{5}{|c|}{ Days after transplanting } & \multicolumn{5}{|c|}{ Days after transplanting } \\
\hline & & & 15 & 30 & 45 & 60 & 75 & 15 & 30 & 45 & 60 & 75 & 15 & 30 & 45 & 60 & 75 \\
\hline \multirow[t]{8}{*}{$\mathrm{A}_{1}$} & $\mathrm{~T}_{1}$ & $\mathrm{~N}_{0}$ & $49.01 \mathrm{fg}$ & 61.42 & $67.11 \mathrm{f}$ & 77.02 & 100.66 & $3.33 \mathrm{ef}$ & $5.33 \mathrm{ef}$ & $6.33 \mathrm{f}$ & $7.67 \mathrm{~g}$ & $8.33 \mathrm{f}$ & 1.40 & 2.24 & $3.11 \mathrm{~cd}$ & $4.79 \mathrm{f}$ & $6.36 \mathrm{~g}$ \\
\hline & & $\mathrm{N}_{1}$ & $50.89 \mathrm{efg}$ & 69.47 & 75.60de & 85.13 & 108.75 & $6.00 \mathrm{a}$ & $8.00 \mathrm{a}$ & $10.67 \mathrm{a}$ & $12.67 \mathrm{a}$ & $15.67 \mathrm{a}$ & 0.85 & 1.97 & $2.86 \mathrm{~d}$ & $5.84 \mathrm{def}$ & $10.71 \mathrm{ab}$ \\
\hline & & $\mathrm{N}_{2}$ & $50.71 \mathrm{fg}$ & 66.27 & $77.23 \mathrm{cde}$ & 84.31 & 106.67 & $5.00 \mathrm{a}-\mathrm{d}$ & $7.00 \mathrm{a}-\mathrm{d}$ & $9.00 \mathrm{bc}$ & $11.00 \mathrm{bc}$ & $14.00 \mathrm{bc}$ & 0.71 & 2.23 & $3.13 \mathrm{~cd}$ & $6.87 \mathrm{a}-\mathrm{d}$ & $11.13 \mathrm{ab}$ \\
\hline & $\mathrm{T}_{2}$ & $\mathrm{~N}_{0}$ & $45.71 \mathrm{~g}$ & 62.52 & $67.32 f$ & 77.40 & 103.17 & $3.00 \mathrm{f}$ & $5.00 \mathrm{f}$ & $6.33 \mathrm{f}$ & $7.67 \mathrm{~g}$ & $9.33 \mathrm{f}$ & 0.84 & 2.22 & $3.24 \mathrm{a}-\mathrm{d}$ & $6.09 \mathrm{cde}$ & 8.94cde \\
\hline & & $\mathrm{N}_{1}$ & $57.01 \mathrm{a}-\mathrm{d}$ & 69.33 & $80.93 \mathrm{abc}$ & 76.00 & 109.37 & $4.67 \mathrm{a}-\mathrm{e}$ & $6.67 \mathrm{a}-\mathrm{e}$ & $7.67 \mathrm{c}-\mathrm{f}$ & $10.67 \mathrm{bcd}$ & $13.67 \mathrm{bcd}$ & 1.07 & 2.30 & $3.28 \mathrm{a}-\mathrm{d}$ & $6.86 \mathrm{a}-\mathrm{d}$ & $10.33 b c$ \\
\hline & & $\mathrm{N}_{2}$ & $49.66 \mathrm{fg}$ & 64.87 & $77.27 \mathrm{cde}$ & 75.67 & 106.37 & $4.33 b-f$ & $6.33 b-f$ & $8.33 b-e$ & $9.67 \mathrm{c}-\mathrm{f}$ & $12.67 \mathrm{cde}$ & 0.77 & 1.94 & $2.83 \mathrm{~d}$ & $6.91 \mathrm{a}-\mathrm{d}$ & $11.29 \mathrm{ab}$ \\
\hline & $\mathrm{T}_{3}$ & $\mathrm{~N}_{0}$ & $53.92 b-f$ & 65.27 & $74.93 \mathrm{e}$ & 80.00 & 107.60 & $4.00 \mathrm{c}-\mathrm{f}$ & $6.00 \mathrm{c}-\mathrm{f}$ & $7.00 \mathrm{ef}$ & $8.33 \mathrm{fg}$ & $11.33 \mathrm{e}$ & 0.90 & 2.67 & $3.64 \mathrm{abc}$ & $7.52 \mathrm{ab}$ & $11.77 \mathrm{ab}$ \\
\hline & & $\mathrm{N}_{1}$ & $51.34 \mathrm{ef}$ & 70.87 & $84.33 \mathrm{ab}$ & 79.27 & 108.77 & $5.67 \mathrm{ab}$ & $7.67 \mathrm{ab}$ & $9.67 \mathrm{ab}$ & $11.67 \mathrm{ab}$ & $14.67 \mathrm{ab}$ & 0.93 & 2.86 & $3.94 \mathrm{a}$ & $6.37 \mathrm{bcd}$ & $9.13 \mathrm{~cd}$ \\
\hline \multirow[t]{10}{*}{$\mathrm{A}_{2}$} & & $\mathrm{~N}_{2}$ & $45.49 \mathrm{~g}$ & 63.07 & $68.60 \mathrm{f}$ & 76.73 & 103.47 & $3.67 \mathrm{def}$ & $5.67 \mathrm{def}$ & $7.67 \mathrm{c}-\mathrm{f}$ & $9.67 \mathrm{c}-\mathrm{f}$ & $12.67 \mathrm{cde}$ & 0.73 & 2.21 & $3.16 \mathrm{bcd}$ & 7.32abc & $11.43 \mathrm{ab}$ \\
\hline & $\mathrm{T}_{1}$ & $\mathrm{~N}_{0}$ & $51.91 \mathrm{def}$ & 69.73 & $75.00 \mathrm{e}$ & 76.80 & 103.73 & $4.00 \mathrm{c}-\mathrm{f}$ & $6.00 \mathrm{c}-\mathrm{f}$ & 7.33def & $9.33 \mathrm{def}$ & $12.33 \mathrm{de}$ & 0.82 & 2.03 & $3.03 \mathrm{~cd}$ & $5.90 \mathrm{def}$ & 7.53efg \\
\hline & & $\mathrm{N}_{1}$ & $49.15 \mathrm{fg}$ & 66.67 & 77.50 cde & 78.67 & 108.77 & $3.67 \mathrm{def}$ & $5.67 \mathrm{def}$ & $7.67 \mathrm{c}-\mathrm{f}$ & $9.67 \mathrm{c}-\mathrm{f}$ & $12.67 \mathrm{cde}$ & 0.89 & 1.98 & $3.08 \mathrm{~cd}$ & $7.57 \mathrm{ab}$ & $11.68 \mathrm{ab}$ \\
\hline & & $\mathrm{N}_{2}$ & $48.93 \mathrm{fg}$ & 66.80 & $77.00 \mathrm{cde}$ & 78.93 & 109.73 & $4.33 b-f$ & $6.33 b-f$ & $8.33 b-e$ & $10.33 \mathrm{~b}-\mathrm{e}$ & $13.33 \mathrm{bcd}$ & 0.85 & 1.94 & $2.94 \mathrm{~cd}$ & $5.13 \mathrm{ef}$ & $7.43 \mathrm{fg}$ \\
\hline & $\mathrm{T}_{2}$ & $\mathrm{~N}_{0}$ & $56.22 \mathrm{~b}-\mathrm{e}$ & 65.00 & $75.50 \mathrm{de}$ & 79.27 & 104.27 & $3.67 \mathrm{def}$ & $5.67 \mathrm{def}$ & $8.33 b-e$ & $9.33 \mathrm{def}$ & $11.33 \mathrm{e}$ & 1.45 & 2.92 & $3.48 \mathrm{a}-\mathrm{d}$ & $6.15 \mathrm{cde}$ & $8.29 \mathrm{def}$ \\
\hline & & $\mathrm{N}_{1}$ & $57.48 \mathrm{abc}$ & 69.23 & $78.30 \mathrm{cde}$ & 76.70 & 105.83 & $6.00 \mathrm{a}$ & $8.00 \mathrm{a}$ & $9.33 \mathrm{ab}$ & $11.33 \mathrm{ab}$ & $14.33 \mathrm{ab}$ & 0.77 & 2.53 & $3.94 a$ & $7.87 \mathrm{a}$ & $11.99 \mathrm{a}$ \\
\hline & & $\mathrm{N}_{2}$ & $53.31 \mathrm{c}-\mathrm{f}$ & 64.20 & $75.30 \mathrm{e}$ & 75.20 & 110.73 & $5.33 \mathrm{abc}$ & 7.33abc & $9.33 \mathrm{ab}$ & $11.33 \mathrm{ab}$ & $13.33 \mathrm{bcd}$ & 0.87 & 2.20 & $3.22 \mathrm{a}-\mathrm{d}$ & $6.04 \mathrm{de}$ & $8.95 \mathrm{cde}$ \\
\hline & $\mathrm{T}_{3}$ & $\mathrm{~N}_{0}$ & $54.41 \mathrm{~b}-\mathrm{f}$ & 69.27 & $80.03 \mathrm{bcd}$ & 77.30 & 108.73 & $3.67 \mathrm{def}$ & $5.67 \mathrm{def}$ & $7.67 \mathrm{c}-\mathrm{f}$ & 9.00efg & $11.33 \mathrm{e}$ & 1.02 & 2.32 & $3.27 \mathrm{a}-\mathrm{d}$ & $6.92 \mathrm{a}-\mathrm{d}$ & $11.24 \mathrm{ab}$ \\
\hline & & $\mathrm{N}_{1}$ & $61.62 \mathrm{a}$ & 67.67 & $84.50 \mathrm{a}$ & 79.37 & 108.23 & $4.67 \mathrm{a}-\mathrm{e}$ & $6.67 a-e$ & $9.33 \mathrm{ab}$ & $11.33 \mathrm{ab}$ & $14.33 \mathrm{ab}$ & 1.73 & 2.33 & $3.22 \mathrm{a}-\mathrm{d}$ & $6.77 \mathrm{a}-\mathrm{d}$ & $10.63 \mathrm{ab}$ \\
\hline & & $\mathrm{N}_{2}$ & $58.77 \mathrm{ab}$ & 73.00 & $81.33 \mathrm{abc}$ & 77.77 & 105.80 & $5.33 \mathrm{abc}$ & 7.33abc & $8.67 \mathrm{bcd}$ & $10.67 \mathrm{bcd}$ & $13.67 \mathrm{bcd}$ & 1.43 & 2.84 & $3.88 \mathrm{ab}$ & 7.06a-d & $10.88 \mathrm{ab}$ \\
\hline CV (\%) & & & 5.44 & 5.72 & 3.12 & 3.63 & 3.62 & 12.19 & 12.56 & 9.66 & 7.98 & 6.46 & 9.69 & 16.96 & 11.72 & 9.7 & 8.12 \\
\hline $\begin{array}{l}\text { Level of } \\
\text { significa } \\
\text { nce }\end{array}$ & & & $* *$ & NS & *** & NS & NS & $*$ & $*$ & $*$ & $*$ & $* *$ & NS & NS & $*$ & $*$ & $*$ \\
\hline
\end{tabular}

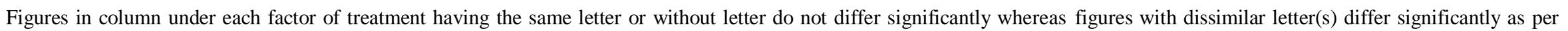

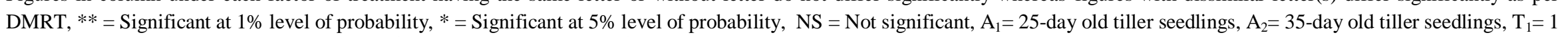
tiller seedlings hill ${ }^{-1}, \mathrm{~T}_{2}=3$ tiller seedlings hill ${ }^{-1}, \mathrm{~T}_{3}=5$ tiller seedlings hill ${ }^{-1}, \mathrm{~N}_{0}=$ control, $\mathrm{N}_{1}=1.8 \mathrm{~g} \mathrm{USG}, \mathrm{N}_{2}=2.7 \mathrm{~g}$ USG. 


\section{Number of tiller hill ${ }^{-1}$}

Number of tiller hill ${ }^{-1}$ was not significantly affected by age of tiller seedlings hill $^{-1}$, number of tiller seedlings hill ${ }^{-1}$ and USG application at all date of observations (Table 1). Hasanuzzaman et al. (2009) reported that application of granular urea produced remarkable number of tillers due to its higher application rate compared to USG but in this case 3 splits produced significantly more tillers than 2 splits. Rana et al. (1989) observed similar results. Hasan (2007) conducted an experiment during the aman season of 2006 and recorded the increased number of tillers hill ${ }^{-1}$ with increased nitrogen levels USG. Rama et al. (1989) mentioned that the number of panicles $\mathrm{m}^{-2}$ increased significantly when nitrogen level increased from 40 to $120 \mathrm{~kg} \mathrm{~N} \mathrm{ha}^{-1}$ as USG. Alam (2002) observed that total tillers hill ${ }^{-1}$ and effective tillers hill ${ }^{-1}$ increased significant with the increase of level of USG, when USG was applied as one, two, three and four granules per 4 hills during the boro season. Singh and Mahapatra (1989) also observed that number of panicles $\mathrm{m}^{-2}$ were significantly higher when USG was applied at $90 \mathrm{~kg} \mathrm{ha}^{-1}$ as deep placement than split application of urea. Singh and Singh (1986) worked with different levels of nitrogen as USG@27.54 and $87 \mathrm{~kg} \mathrm{ha}^{-1}$. They reported that number of tillers $\mathrm{m}^{-2}$ increased with increasing nitrogen fertilizer. Hasanuzzaman et al. (2009) reported that deep placement of USG showed highest number of tillers might be due to little loss of $\mathrm{N}$ from soil and slowly releasing process.

Number of tiller hill ${ }^{-1}$ was significantly influenced by the interaction between the age of tiller seedlings and number of tillers seedlings hill ${ }^{-1}$ at 15, 30, 45 and 60 DAT. At 15, 30, 45 and 60 DAT the highest number of tillers hill ${ }^{-1}$ was found in 35-day old tiller seedlings by transplanting 3 tiller seedlings hill $^{-1}$ and the lowest number of tillers hill ${ }^{-1}$ was found in 25day old tiller seedlings by transplanting 3 tiller seedlings hill $^{-1}$ (Table 2). Number of tillers hill $^{-1}$ was significantly influenced by the interaction between the age of tiller seedlings and USG application within all the dates of observations. At $15,30,45,60,75$ DAT the highest number of tillers hill ${ }^{-1}$ was found in 25-day old tiller seedlings when $1.8 \mathrm{~g}$ USG was applied and the lowest number of tillers hill ${ }^{-1}$ was found in 25 day old tiller seedlings when USG was not applied at (Table 3 ). Number of tillers hill ${ }^{-1}$ was significantly influenced by the interaction between number of tiller seedlings hill ${ }^{-1}$ and application of USG at 30 and 45 DAT. At 30 and 45 DAT the highest number of total tiller hill ${ }^{-1}$ was found by transplanting 5 tiller seedlings hill $^{-1}$ when
$1.8 \mathrm{~g}$ USG was applied and the lowest number of tiller hill ${ }^{-1}$ was found by transplanting 1 tiller seedlings hill ${ }^{-1}$ when USG was not applied (Table 4). Number of tillers hill ${ }^{-1}$ was significantly influenced by the interaction between age of tiller seedlings, number of tiller seedlings hill ${ }^{-1}$ and USG application at all the dates of observations. At 15 and 30 DAT the highest number of total tiller hill ${ }^{-1}$ was found in 35-day old tiller seedling by transplanting 3 tiller seedlings hill ${ }^{-1}$ with the application of $1.8 \mathrm{~g}$ USG and the lowest number of total tiller hill ${ }^{-1}$ was found in 25-day old tiller seedlings by transplanting 3 tiller seedlings hill $^{-1}$ without application of USG. At 45, 60 and 75 the highest number of total tiller hill $^{-1}$ was found in 25-day old tiller seedling by transplanting 1 tiller seedling hill ${ }^{-1}$ with the application of $1.8 \mathrm{~g}$ USG and the lowest number of total tiller hill ${ }^{-1}$ was found in 25-day old tiller seedlings by transplanting 1 tiller seedling hill $^{-1}$ without application of USG (Table 5).

\section{Total dry matter production}

There was significant effect of age of tiller seedlings on total dry matter production hill $^{-1}$ at 15, 45 and 75 days after transplanting (Table 1). The highest total dry matter production was found when 25-day old tiller seedlings were transplanted while dry matter production was reduced when 35-day old tillers were transplanted (Figure 2). Total dry matter production hill $^{-1}$ was significantly affected by number of tiller seedlings hill ${ }^{-1}$ at 30, 45, 60 and 75 DAT. The highest total dry matter production hill $^{-1}$ was found at all dates when 5 tiller seedlings were transplanted hill $^{-1}$. The lowest one was found at all dates of observations when 1 tiller seedling was transplanted hill $^{-1}$. The intermediate total dry matter yield was recorded while 3 tiller seedlings were transplanted. Total dry matter production hill ${ }^{-1}$ was found to increase with increasing the number of tiller seedlings hill $^{-1}$. This finding was supported by Hossain et al. (2011). They obtained the highest total dry matter when 6 tiller seedlings were transplanted hill ${ }^{-1}$ which was as good as 6 tiller seedlings transplanted hill $^{-1}$ and the lower one also recorded with 2 tiller seedlings were transplanted hill ${ }^{-1}$. Sarkar et al. (2002) reported that maximum total dry matter was obtained in intact hill compared to separated hill (Table 1). Total dry matter production hill ${ }^{-1}$ was significantly affected at 60 and 75 DAT due to the effect of USG application. The highest total dry matter production hill $^{-1}$ was found at all dates when $1.8 \mathrm{~g}$ USG was applied and the lowest 
was found at all date of observations when USG was not applied (Table 1). Roy et al. (1990) and Mamin et al. (1999) reported that straw yield always significantly higher in intact mother hills than splitted treatments which might be due to higher plant height, more tillers unit ${ }^{-1}$ area and undisturbed vegetative growth. Total dry matter production hill $^{-1}$ was significantly influenced by the interaction between the age of tiller seedlings and number of tiller seedlings hill ${ }^{-1}$ at 15 DAT. The highest total dry matter production hill ${ }^{-1}$ was found in 35-day old tiller seedlings by transplanting 5 tiller seedlings hill $^{-1}$ and the lowest total dry matter production hill $^{-1}$ was found in 35-day old tiller seedlings by transplanting 5 tiller seedlings hill $^{-1}$ followed by 35-day old tiller seedling by transplanting 3 seedlings hill ${ }^{-1}$ and 25-day old tiller seedlings by transplanting 1 tiller seedling hill $^{-1}$ (Table 2). Total dry matter production hill ${ }^{-1}$ was significantly influenced by the interaction between the age of tiller seedlings and USG application at 45, 60 and 75 DAT. At 60 and 75 DAT the highest total dry matter production hill ${ }^{-1}$ was found in 35-day old tiller seedlings when $1.8 \mathrm{~g}$ USG was applied and the lowest total dry matter production hill ${ }^{-1}$ was found in 35-day old tiller seedlings when $2.7 \mathrm{~g}$ USG was applied (Table 3). Total dry matter production hill ${ }^{-1}$ was significantly influenced by the interaction between number of tiller seedlings hill ${ }^{1}$ and USG application at 60 and 75 DAT. At 60 and 75 DAT the highest total dry matter production hill-1 was found by transplanting 3 tiller seedlings hill $^{-1}$ when $1.8 \mathrm{~g}$ USG was applied and the lowest total dry matter production hill $^{-1}$ was found by transplanting 1 tiller seedlings hill $^{-1}$ when USG was not applied (Table 4). Total dry matter production hill ${ }^{-1}$ was significantly influenced by the interaction between age of tiller seedlings, number of tiller seedlings hill $^{-1}$ and USG application at 45, 60 and 75. At 45 DAT the highest total dry matter production hill 1 was found in 25-day old tiller seedling by transplanting 5 tiller seedlings hill $^{-1}$ with the application of $1.8 \mathrm{~g}$ USG which is as like as when transplanting 35-day old tiller seedling by transplanting 3 tiller seedlings hill $^{-1}$ with the application of $1.8 \mathrm{~g}$ USG and the lowest total dry matter production hill ${ }^{-1}$ was found in 25-day old tiller seedlings by transplanting 1 tiller seedling hill $^{-1}$ with the application of $1.8 \mathrm{~g}$ USG. At 60 and 75 DAT the highest total dry matter production hill ${ }^{-1}$ was found in 35-day old tiller seedling by transplanting 3 tiller seedlings hill $^{-1}$ with the application of $1.8 \mathrm{~g}$ USG and the lowest total dry matter production hill ${ }^{-1}$ was found in 25-day old tiller seedlings by transplanting 1 tiller seedling hill ${ }^{-1}$ without application of USG (Table 5).

\section{Conclusion}

From the findings of the experiment it can be concluded that tallest plant and total dry matter production hill ${ }^{-1}$ could be obtained in transplant Aman rice planted by transplanting 25-day old tiller seedlings with 5 tiller seedlings hill ${ }^{-1}$. Application of $1.8 \mathrm{~g}$ urea super granules appeared as the promising practice in respect of tallest plant and total dry matter production hill ${ }^{-1}$.

\section{References}

Akhter, S. 1999. Effect of row-spacing and urea super granule on the yield and yield contributing characters of T. aman rice. MS (Ag) Thesis in Agronomy, BAU. Mymensingh. 33p.

Alam, B.M.R. 2002. Effect of urea super granule on the growth and yield of three varieties of boro rice. MS (Ag.) Thesis, Dept. Agron., Bangladesh Agril. Univ., Mymensigh.119 p.

Alim, A.; Sen, J. L.; Ullah, M. T. and Chowdhury, M. A. 1962. Review of half a century of rice research in east Pakistan. Govt. of East Pakistan, EPG press Dhaka, pp: 119.

Biswas, P. K. and Salokhe, V. M. 2001. Effects of planting date, intensity of tiller separation and plant density on the yield of transplanted rice. The journal of Agricultural science, 137: 279287.

Hasan, S. M. 2007. Effect of level of urea super granules on the performance of T. aman rice. M. Sc. Ag. Thesis in Agronomy, BAU, Mymensingh.

Hasanuzzaman, M.; Nahar,K.; Alam, M. M.; Hossain, M. Z. and Islam, M. R. 2009. Response of transplant aman rice to different application methods of urea fertilizer. International Journal of Agriculture, 1 (1): 01-05

Hossain, M. A.; Sarkar, M. A. R. and Paul, S. K. 2011. Growth analysis of late transplant aman rice (cv. BR 23) raised from tiller seedlings. Libyan Aric. Res. Cen. J. Intl., 2 (6): 265-273.

Hossain, M. M. and Haque, M. Z. 1990. Seedlings age and density effect on basal tiller survival and yield of transplant deep water rice.[Cited from Rice Abst. 13 (5): 250. 
Mamin, M. S. I.; Alam, M. Z.; Ahmed, A. U.; Rashid, M. A.; and Jameel, F. 1999. Effect of splitting tillers on the yield and yield components of transplanted Aman rice. Ann. Bangla. Agric., 9: 1-9.

Mishra, B. K.; Mishra., Das, A. K. and Jena, D. 2000. Effect of time for urea super granule placement of low land rice. Ann. Res. 20:4, 443-447.

Mollah, M. I. U.; Hossain, S. M. A.; Islam, N. and Miah, M. N. I. 1992. Some aspects of tiller separation in transplant Aman rice. Bangla. Agron. J., 4: 45-49.

Mridha, M. A.; Nasiruddin J. M. and Siddique, S. B. 1991. Tiller separation on yield and area covered in rice. Proc. of the $16^{\text {th }}$ Ann. BAAS conf. held on 5-7 July 191, Dhaka pp: 67.

Rahman, M. A. 2003. Effect of levels of urea super granules and depth of placement on the growth and yield of transplant amanrice. MS (Ag.) Thesis, Dept. Agron., Bangladesh Agril, Univ., Mymensingh. 100 p.

Rana, S.; Reddy, G. and Reddy, K.1989. Effect of levels and sources of nitrogen on rice. Indian J. Agron., 34: 435-436.

Rama, S.; Reddy, G. and Reddy, K. 1989. Effect of levels and sources of nitrogen on rice. Indian J. Agron., 34: 435-436.

Roy, S. K.; Biswas P. K. and Quasem, A.1990. Effects of tiller removal and replanted tillers on the yield of the main and the subsequent rice crops. Bangla. J. Agric., 15: 11-18.
Sarkar, M. A. R.; Paul, S. K. and Hossain, M. A. 2011. Effect of row arrangement, age of tiller seedlings and number of tiller seedlings hill ${ }^{-1}$ on performance of transplant aman rice. The Journal of Agricultural Science, 6 (2): 59-68.

Sarkar, M. A. R.; Paul, S. K. and Ahmed. M. 2002. Effect of row arrangement and tiller separation on the growth of transplant Aman rice. Pakistan Journal of Biological Sciences, 5(4): 504-506.

Siddique, S. B.; Mazid, M. A.; Mamun, M. A.; Ahmed, K. U.; Jabbar, M. A.; Mridha, A. J.; Ali, M. G.; Chowdhury, A. A.; Roy, B. C.; Hafiz, M. A.; Biswas, J. C. and Islam, M. S. 1991. Cultural practices for modern rice cultivation under low land ecosystems. Proceedings of workshop on experiences with modern rice cultivation in Bangladesh held in 23-25 April, 1991 at BRRI, Gazipur.

Singh, S. P. and Singh, M. P. 1986. Response of dwarf rice cv. Jaya to different rates, methods and forms of urea materials. Environ. And Ecol., 15 (3): 612-613.

Singh, I. C. and Mahapatra, I. C. 1989. Economics of use. Pert. Marketing Newsl., 5(12):1-17. 